\title{
Precise Measurements of the Kilohertz Quasi-Periodic Oscillations in 4U 1728-34
}

\author{
Mariano Méndez ${ }^{1,2}$, Michiel van der Klis ${ }^{1}$
}

\begin{abstract}
We have analyzed seventeen observations of the low-mass X-ray binary and atoll source 4U 1728-34, carried out by the Rossi X-ray Timing Explorer in 1996 and 1997. We obtain precise measurements of the frequencies of the two simultaneous kilohertz quasi-periodic oscillations ( $\mathrm{kHz} \mathrm{QPOs})$ in this source. We show that the frequency separation between the two QPO, $\Delta \nu$, is always significantly smaller than the frequency of the nearly-coherent oscillations seen in this source during X-ray bursts, even at the lowest inferred mass accretion rate, when $\Delta \nu$ seems to reach its maximum value. We also find that $\Delta \nu$ decreases significantly, from $349.3 \pm 1.7 \mathrm{~Hz}$ to $278.7 \pm 11.6 \mathrm{~Hz}$, as the frequency of the lower frequency kHz QPO increases from 615 to $895 \mathrm{~Hz}$. This is the first time that variations of the $\mathrm{kHz} \mathrm{QPO}$ peak separation are measured in a source which shows nearly-coherent oscillations during bursts.
\end{abstract}

Subject headings: accretion, accretion disks — stars: neutron — stars: individual (4U 1728-34) - X-rays: stars

\footnotetext{
${ }^{1}$ Astronomical Institute "Anton Pannekoek", University of Amsterdam and Center for High-Energy Astrophysics, Kruislaan 403, NL1098 SJ Amsterdam, the Netherlands

${ }^{2}$ Facultad de Ciencias Astronómicas y Geofísicas, Universidad Nacional de La Plata, Paseo del Bosque S/N, 1900 La Plata, Argentina
} 


\section{Introduction}

Quasi-periodic oscillations at frequencies near 1 kilohertz (kHz QPOs) are now known to be a common phenomenon among low mass X-ray binaries (LMXBs). Since the beginning of the Rossi X-ray Timing Explorer (RXTE) mission $\mathrm{kHz}$ QPOs have been discovered in the persistent flux of nineteen LMXBs (see van der Klis 1998 for a review). In almost all cases the power density spectra of these sources show twin $\mathrm{kHz}$ peaks that, as a function of time, gradually move up and down in frequency, typically over a range of several hundred $\mathrm{Hz}$.

The dependence of the QPO frequencies upon Xray flux is complex. One example is $4 \mathrm{U}$ 1608-52 (Méndez et al. 1999): While on time scales of hours frequency and X-ray flux are well correlated, at epochs separated by days to weeks the QPOs span the same range of frequencies even if the average flux is different by a factor of 3 or more (see also Ford et al. 1997; Zhang et al. 1998). Ford et al. (1997) have shown that in $4 \mathrm{U} 0614+09$ the QPO frequency is better correlated to the flux of the soft ("blackbody") component in its X-ray spectrum than to the total Xray flux, while Kaaret et al. (1998) found, both for $4 \mathrm{U} 0614+09$ and $4 \mathrm{U} 1608-52$, a good correlation between the QPO frequency and the photon index of the power-law component in the X-ray spectrum. Méndez et al. (1999) showed that QPO frequency is very well correlated to the position of the source on the colorcolor diagram, and they concluded that most likely these frequency changes are driven by changes in $\dot{M}$ through the innermost part of the accretion disk, and that there is no one-to-one relationship between the observed X-ray flux and this mass accretion rate.

Initially, data from various sources suggested that the separation $\Delta \nu$ between the twin $\mathrm{kHz}$ peaks remained constant even as the peaks moved up and down in frequency (e.g., Strohmayer et al. 1996). In some sources a third, nearly-coherent, oscillation is detected during type-I X-ray bursts, at a frequency close to $\Delta \nu$, or twice that value (see Strohmayer, Swank, \& Zhang 1998 for a review). These two results suggested that a beat-frequency mechanism was at work (Strohmayer et al. 1996; Miller, Lamb, \& Psaltis 1998), with the third peak being close to the neutron star spin frequency or twice that.

It is now clear that $\Delta \nu$, which in the beat-frequency interpretation is thought to be equal to the spin frequency of the neutron star, is not always constant.
In Sco X-1 (van der Klis et al. 1997), 4U 1608-52 (Méndez et al. 1998d), and 4U 1735-44 (Ford et al. 1998), $\Delta \nu$ slowly decreases as the frequencies of both QPOs increase. In these sources no burst oscillations have been detected so far. In $4 \mathrm{U} 1636-53$ the frequency of the burst oscillations (or half this value) does not exactly match the frequency difference between the kHz QPOs (Méndez, van der Klis, \& van Paradijs 1998a). In this case, however, no evidence was found of any significant change of $\Delta \nu$. So far, no measurements existed of burst oscillations and a varying $\mathrm{kHz}$ peak separation in the same source.

In this paper we present such measurements for the first time. We show that in $4 \mathrm{U}$ 1728-34, the frequency separation between the two simultaneous $\mathrm{kHz}$ QPOs is always lower than the frequency of the burst oscillations, even at low $\mathrm{kHz}$ QPO frequencies, when the peak separation appears to have reached its highest value. We also find that $\Delta \nu$ decreases significantly as the frequencies of the $\mathrm{kHz}$ QPOs increase. As in $4 \mathrm{U}$ $1608-52$, the QPO frequencies in $4 \mathrm{U} 1728-34$ are well correlated to the position of the source on the colorcolor diagram, despite them not being correlated to the X-ray flux.

\section{Observations}

RXTE observed $4 \mathrm{U} 1728-34$ on ten occasions in 1996, between February 15 and March 1, for a total of $199 \mathrm{ks}$ (for a description of these observations see Strohmayer et al. 1996, 1997; Ford \& van der Klis 1998), and seven more times starting UTC 1996 May 3 13:56:00 for $3.9 \mathrm{ks}$, 1997 September 23 23:48:00 for $1.4 \mathrm{ks}$, September 24 09:13:00 for $22.9 \mathrm{ks}$, September 26 12:28:00 for $18.1 \mathrm{ks}$, September 27 09:17:00 for 18.3 ks, September 30 04:31:00 for 20.6 ks, and October 1 06:07:00 for $21.2 \mathrm{ks}$.

Here we only use data recorded by the Proportional Counter Array (PCA) onboard RXTE (Bradt, Rothschild, \& Swank 1993). Besides the two standard modes that are available to all RXTE/PCA observations, data were collected using additional modes with high time, and moderate energy resolution, covering the nominal $2-60 \mathrm{keV}$ energy band of the $R X T E /$ PCA. Part of these data (the 1996 observations) were analyzed previously (Strohmayer et al. 1996, 1997). Their analysis of the $\mathrm{kHz}$ QPOs was based on a simple direct FFT method, while ours uses the "shift-and-add" method (see below).

To estimate the source X-ray intensity and colors 
we measured the count rates every $16 \mathrm{~s}$ in 5 energy bands, $2.0-3.5-6.4-9.7-16.0 \mathrm{keV}$, and $2.0-16.0$ $\mathrm{keV}$, taking into account the gain changes applied to the PCA in March and April 1996. In a few of the observations one or two of the five detectors of the PCA were switched off; we only used the three detectors which were always on to calculate these count rates. We subtracted the background contribution in each band using the standard PCA background model version $2.1 \mathrm{~b}$, and normalized the count rates to 5 detectors. For the timing analysis we used data from all active detectors without energy selection.

\section{Results}

\subsection{Measurement of the frequencies of the QPOs}

We divided the high-time resolution data into segments of $64 \mathrm{~s}$, and calculated a power spectrum for each segment preserving the maximum available Nyquist frequency (8192 or $65536 \mathrm{~Hz}$, depending on the observation). We produced an average power spectrum for each observation and searched it for QPOs at frequencies above $100 \mathrm{~Hz}$.

In the rest of this subsection we only present the results of the analysis of those 1482 data segments in observations where we detected two simultaneous $\mathrm{kHz}$ QPOs in the power spectrum. Here we concentrate on the measurements of the frequency separation $\Delta \nu$ of these QPOs, as other properties of the QPOs have already been reported in previous papers (Strohmayer et al. 1996; Ford \& van der Klis 1998; for the observations that we report here for the first time, the other properties of the QPOs are similar to those described in these two papers).

To measure $\Delta \nu$ we applied the 'shift-and-add' technique advanced by Méndez et al. (1998b). Measuring $\Delta \nu$ in an averaged (non-shifted) power spectrum can introduce a bias that changes the peak separation from its true value because, in general, the two QPOs vary in strength in a different manner as they move in frequency (see for instance Wijnands et al. 1997). This is no longer the case if the individual power spectra are shifted to the frequency of one of the two peaks before they are averaged together. (However, this method can be applied only if one of the QPOs is always strong enough to measure its frequency in each of the individual power spectra.) A second advantage of this method is that longer stretches of data can be used to measure $\Delta \nu$, and not just pieces of data where the QPO frequencies are more or less constant. This, in turn, decreases the errors in the measurements of the frequency separation (see below).

We measured the central frequency of the lower frequency QPO in each segment of $64 \mathrm{~s}$ by fitting a Lorentzian to it (this QPO was well detected in each segment). We then grouped the data in 8 sets of 30 , $58,66,193,371,262,244$, and 258 power spectra, such that the frequency of the QPO did not vary by more than $\sim 50 \mathrm{~Hz}$ within each set (the first 2 sets contain data from 1996 only, whereas the last 2 sets contain data from 1997 only; segments 3 to 6 include data from both epochs). We then shifted the frequency scale of each spectrum to a frame of reference where the position of this peak was constant in time, and we finally averaged the aligned spectra in each set.

We fitted the resulting 8 average power spectra in the range $256-1500 \mathrm{~Hz}$ using a function consisting of a constant, representing the Poisson noise, and two Lorentzians, representing the QPOs. The fits were good, with reduced $\chi^{2} \leq 1.1$ for 1585 degrees of freedom, and the significance of each peak was always $>4 \sigma$. In Figure 1 we plot the frequency difference, $\Delta \nu$, between the higher frequency and the lower frequency QPOs (hereafter the upper and lower QPO, respectively) as a function of the centroid frequency of the lower QPO, $\nu_{\text {low }}$. The solid line indicates the frequency of the oscillations detected during the X-ray bursts, $\nu_{\text {burst }}=363.95 \mathrm{~Hz}$ (Strohmayer et al. 1996, 1998). These results are entirely consistent with those of Strohmayer et al. 1996; Table 1), but our error bars, thanks to the use of the "shift-and-add" technique, are much smaller than theirs.

Because of these small error bars, it is now apparent that $\Delta \nu$ is always smaller than $\nu_{\text {burst }}$. The figure shows that $\Delta \nu$ is consistent with being constant at $\nu_{\text {low }}<800 \mathrm{~Hz}$, but decreases as $\nu_{\text {low }}$ increases above $800 \mathrm{~Hz}$. The data point near $\nu_{\text {low }}=900 \mathrm{~Hz}$ is significantly $(6 \sigma)$ below the average of the other points. If, conservatively, we discard both points at $\nu_{\text {low }}>800 \mathrm{~Hz}$ the average frequency separation becomes $\Delta \nu=349.3 \pm 1.7 \mathrm{~Hz}$ (indicated by the dashed line in Fig. 11), still different from $363.95 \mathrm{~Hz}$ at an $8.6 \sigma$ level. So, even in the range where $\Delta \nu$ appears to have reached its saturation value, it is significantly below the frequency of the burst oscillations. 


\subsection{QPO frequency as a function of inferred $\dot{M}$}

In Figure 2 we show a color-color diagram of $4 \mathrm{U}$ 1728-34, which is shown here for the first time to be typical of those of other atoll sources (Hasinger \& van der Klis 1989). In general, the count rate is observed to increase as indicated by the arrow on the diagram, from the island state (at the upper right part of the diagram) to the banana branch, but the relation between count rate and either of the two colors is much less clean than that between colors. Each point in this diagram represents $128 \mathrm{~s}$ of data. Dots and open circles indicate the segments with and without detectable $\mathrm{kHz}$ QPOs, respectively.

In Figure 3 we show the frequency of the lower QPO, $\nu_{\text {low }}$, as a function of count rate for $4 \mathrm{U} 1728$ 34 . Each point in this figure represents $128 \mathrm{~s}$ of data. The branch in the lower right part of the diagram corresponds to the data presented by Strohmayer et al. (1996) for the lower $\mathrm{kHz}$ QPO. This plot can be compared to those in Aql X-1 (Zhang et al. 1998) and 4U 1608-52 (Méndez et al. 1999). As in those sources, for $4 \mathrm{U} 1728-34$ it is also true that on time scales greater than $\sim 1$ day there is no unique relation between count rate and $\nu_{\text {low }}$.

As in the case of $4 \mathrm{U} 1608-52$ (Méndez et al. 1999), QPO frequencies correlate much better to the position of the source on the color-color diagram than to count rate. In Figure 1 we show $\nu_{\text {low }}$ as a function of the position on the color-color diagram as measured by the parameter $S_{\mathrm{a}}$ (curve length in the color-color plane measured in the direction of the arrow in Fig. 2; see Méndez et al. 1999 for the precise definition of $S_{\mathrm{a}}$ ), for the same intervals shown in Figure 3 (black circles). In this case $S_{\mathrm{a}}=1$ at colors $(0.48,-0.23)$, and $S_{\mathrm{a}}=2$ at $(0.44,-0.34)$. The black squares represent $\nu_{\text {upp }}$, the frequency of the upper $\mathrm{kHz}$ QPO as a function of $S_{\mathrm{a}}$ (we only include here segments where we could measure $\nu_{\text {upp }}$ directly). The grey triangles are measurements from segments in which we only detect one of the $\mathrm{kHz}$ QPOs; however, from the location of each point in this diagram we can determine whether it is the lower or the upper QPO. While the frequency vs. count rate relation (Fig. 2) is complex, there seems to be a neat relation between each QPO frequency and $S_{\text {a }}$ (Fig. 目).

\section{Discussion}

We have for the first time measured the variation of the $\mathrm{kHz}$ QPO peak separation in a source which shows oscillations during X-ray bursts. The result of this measurement is unexpected: even at its saturation level at low inferred $\dot{M}, \Delta \nu$ is still significantly below the frequency of the burst oscillations. Before we discuss this further, we consider what our data imply about the relation between the $\mathrm{kHz}$ QPO frequencies and $\dot{M}$.

Despite the complex relation between frequency and count rate (Fig. 3), in $4 \mathrm{U} 1728-34$ there is a unique relation between frequency and position in the X-ray color-color diagram (Fig. (1), similar to what is observed in $4 \mathrm{U}$ 1608-52 (see Fig. 2, 3, and 4 in Méndez et al. 1999). As in 4U 1608-52, in 4U 1728 34 the frequencies of the QPOs increase with $S_{\mathrm{a}}$, as the source moves from the island to the banana. The presence of the QPOs also correlates with the position in the color-color diagram. This may be a general feature. In atoll sources it has been proposed that $\dot{M}$ increases with $S_{\text {a }}$ from the island to the banana (Hasinger \& van der Klis 1989), even though X-ray flux is not monotonically related to $S_{\text {a }}$ (van der Klis et al. 1990; van der Klis 1994; Prins \& van der Klis 1997). Our proposed interpretation is that the observed changes in the frequency of the $\mathrm{kHz}$ QPOs in LMXBs are driven by changes in $\dot{M}$ through the innermost part of the accretion disk, and that while the observed X-ray flux is not a good indicator of this mass accretion rate, the $\mathrm{X}$-ray colors track it much better. Possible reasons for this include anisotropies in the emission, redistribution of some of the radiation over unobserved energy ranges, and matter that flows onto the neutron star in a more radial inflow, or away from it in a jet.

We have found that in $4 \mathrm{U} 1728-34$ the frequency separation between the two simultaneous $\mathrm{kHz}$ QPOs, $\Delta \nu$, is not consistent with being equal to the frequency of the burst oscillations. This is the second source, after $4 \mathrm{U}$ 1636-53, for which $\Delta \nu$ does not match $\nu_{\text {burst }}$. Furthermore, in $4 \mathrm{U} 1728-34 \Delta \nu$ is not constant, but it decreases as the frequency of the lower $\mathrm{kHz}$ QPO increases. This is the first source in which the three oscillations are seen simultaneously, and in which the separation between the frequencies of the $\mathrm{kHz}$ QPOs is observed to change.

It has been proposed that the upper $\mathrm{kHz} \mathrm{QPO}$ at $\nu_{\text {upp }}$ represents the Keplerian frequency of the ac- 
creting material in orbit around the neutron star at some preferred radius (van der Klis et al. 1996), while the lower QPO, at $\nu_{\text {low }}$, is produced by the beating of $\nu_{\text {upp }}$ with the spin frequency of the neutron star, $\nu_{\text {spin }}$, which has been identified with $\nu_{\text {burst }}$ (or half that; Strohmayer et al. 1996; Miller et al. 1998). If this interpretation is correct, $\Delta \nu$ equals $\nu_{\text {spin }}$ and should remain constant. The neutron star spin can not change by $\sim 20-30 \%$ on timescales of days to months.

There are now four sources for which $\Delta \nu$ has been observed to vary: Sco X-1 (van der Klis et al. 1997), 4U 1608-52 (Méndez et al. 1998d, 4U 1735-44 (Ford et al. 1998), and $4 \mathrm{U} 1728-34$. It has been argued that these variations can be attributed to the near-Eddington mass accretion rate: White \& Zhang (1997) propose a $35 \%$ expansion of the neutron star photosphere with conservation of angular momentum, and F. K. Lamb (1996, private communication) suggests that the height of the inner disk increases, and the different values of $\Delta \nu=\nu_{\text {upp }}-\nu_{\text {low }}$ reflect different values of $\nu_{\text {upp }}$ at different heights in the disk.

Contrary to what we observe in $4 \mathrm{U}$ 1728-34, these explanations imply that $\Delta \nu$ should approach $\nu_{\text {spin }}$ when, at low $\dot{M}$, the $\mathrm{kHz}$ QPOs move to lower frequencies. Our results show that in $4 \mathrm{U} 1728-34, \Delta \nu$ is still significantly below $\nu_{\text {burst }}$ at the lowest inferred $\dot{M}$ values. This seems to rule out the simple beatfrequency interpretation of the $\mathrm{kHz}$ QPOs in LMXBs, and some of the modifications introduced to explain the results of a variable $\Delta \nu$.

Recent ideas allowing the observed beat-frequency to deviate slightly from the true one (F. K. Lamb 1998, private communication), as well as proposals discarding one or more elements of the basic beatfrequency picture, but still predicting definite relations between the observed frequencies (e.g., Stella \& Vietri 199; Titarchuk, Lapidus, \& Muslimov 1998) therefore merit careful consideration.

We are thanked to Will Zhang for for his comments that helped us to improve the original manuscript. This work was supported in part by the Netherlands Organization for Scientific Research (NWO) under grant PGS 78-277, by the Netherlands Foundation for research in astronomy (ASTRON) under grant 78176-017, and by the Netherlands Research School for Astronomy (NOVA). MM is a fellow of the Consejo Nacional de Investigaciones Científicas y Técnicas de la República Argentina. This research has made use of data obtained through the High Energy Astrophysics Science Archive Research Center Online Service, provided by the NASA/Goddard Space Flight Center.

\section{REFERENCES}

Bradt, H. V., Rothschild, R. E., \& Swank, J. 1993, A\&AS, 97, 355

Ford, E., et al. 1997, ApJ, 486, L47

Ford, E. \& van der Klis, M. 1998, ApJ, 506, L39

Ford, E. C., van der Klis, M., van Paradijs, J., Méndez, M., Wijnands, R., \& Kaaret, P. 1998, ApJ, 508, L155

Hasinger, G., \& van der Klis, M. 1989, A\&A, 225, 79

Kaaret, P., Yu, W., Ford, E. C., \& Zhang, N. S. 1998, ApJ, 497 L93

Méndez, M., van der Klis, \& van Paradijs, J. 1998a, ApJ, 506, L117

Méndez, M., van der Klis, M., van Paradijs, J., Lewin, W. H. G., Vaughan, B. A., Kuulkers, E., Zhang, W., Lamb, F. K., \& Psaltis, D. 1998b, ApJ, 494, L65

Méndez, M., van der Klis, M., Wijnands, R., Ford, E. C., van Paradijs, J., \& Vaughan, B. A. 1998c, ApJ, 505, L23

Méndez, M., van der Klis, M., Ford, E. C., Wijnands, R., \& van Paradijs, J. 1999, ApJ, 511, L49

Miller, M. C., Lamb, F. K., Psaltis, D. 1998, ApJ, 791

Prins, S., \& van der Klis, M. 1997, A\&A, 319, 498

Stella. L. \& Vietri, M. 1999, Phys. Rev. Lett., in press astro$\mathrm{ph} / 9812124$ )

Strohmayer, T. E., Swank, J. H., \& Zhang, W. 1998, ApJ, 503, L147

Strohmayer, T. E., Zhang, W., Swank, J. H., Smale, I., Titarchuk, L., Day, C. \& Lee, U. 1996, ApJ, 469, L9

Strohmayer, T. E., Zhang, W., \& Swank, J. H. 1997, ApJ, 487, L77

Titarchuk, L., Lapidus, I., Muslimov, A. \& 1998, ApJ, 499, 315

van der Klis, M. 1994, ApJS, 92, 511

van der Klis, M. 1998, in Proc. of the 3rd William Fairbank Meeting, Rome June 29 - July 41998 astro-ph/9812395

van der Klis, M., Hasinger, G., Damen, E., Penninx, W., van Paradijs, J., \& Lewin, W. H. G. 1990, ApJ, 360, L19

van der Klis, M., et al. 1996, IAU Circ.6319

van der Klis, M., Wijnands, R. A. D., Horne, K., \& Chen, W. 1997, ApJ, 481, L97

White, N. E., \& Zhang, W. 1997, ApJ, 490, L87

Wijnands, R. A. D., van der Klis, M., van Paradijs, J., Lewin, W. H. G., Lamb, F. K., Vaughan, B. A., \& Kuulkers, E. 1997 ApJ, 479, L141

Zhang, W., Jahoda, K., Kelley, R. L., Strohmayer, T. E., Swank, J. H., \& Zhang, N. 1998, ApJ, 495, L9

This 2-column preprint was prepared with the AAS LATEX macros $\mathrm{v} 4.0$. 


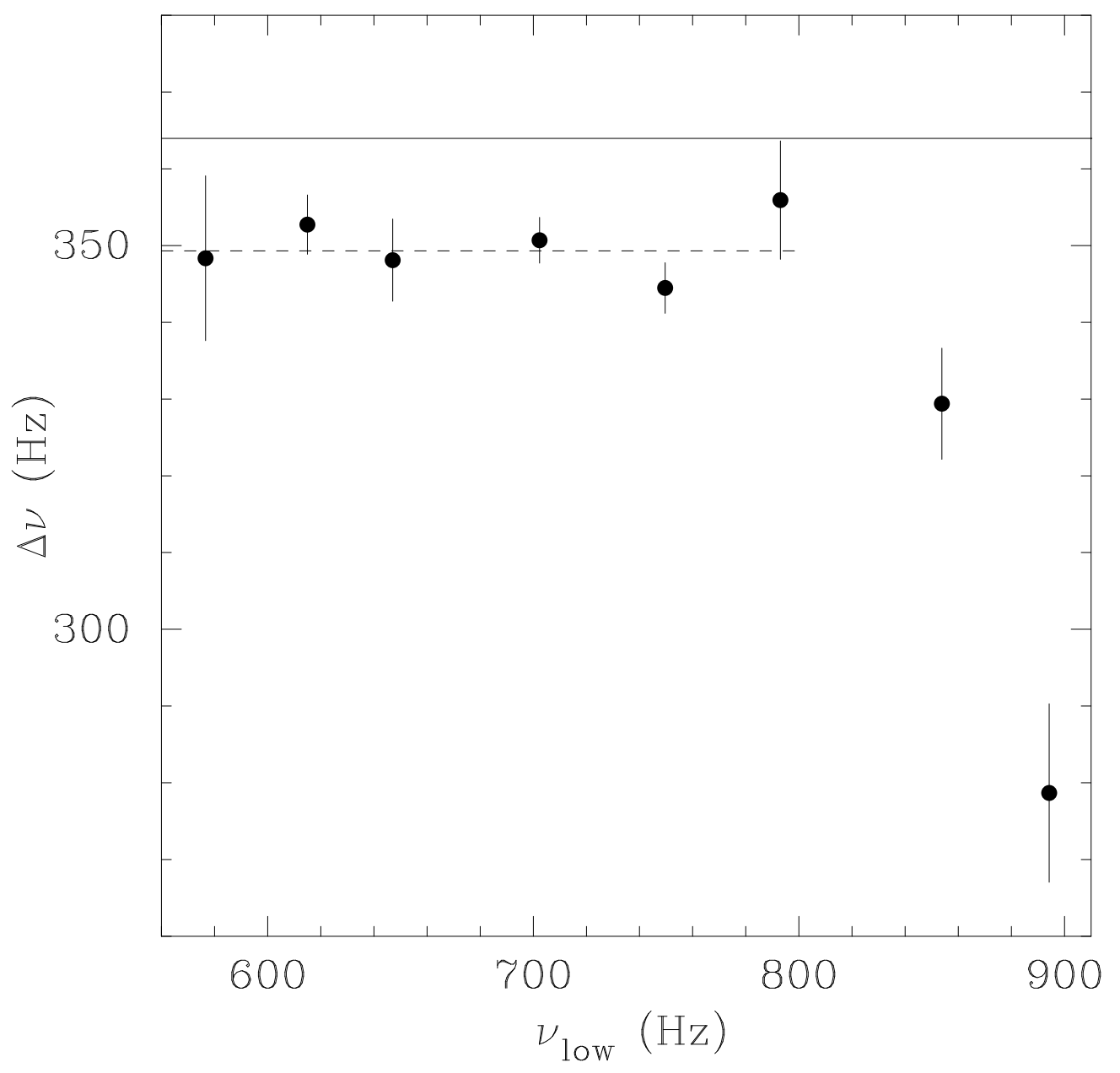

Fig. 1.- The frequency separation between the upper and the lower QPO peak as a function of the frequency of the lower QPO for $4 \mathrm{U} 1728-34$ (circles). The solid line indicates the frequency of the burst oscillations at 363.95 $\mathrm{Hz}$ (Strohmayer et a. 1996, 1998). The dashed line indicates the average value of $\Delta \nu(349.3 \mathrm{~Hz})$ for the first 6 points (see text). 


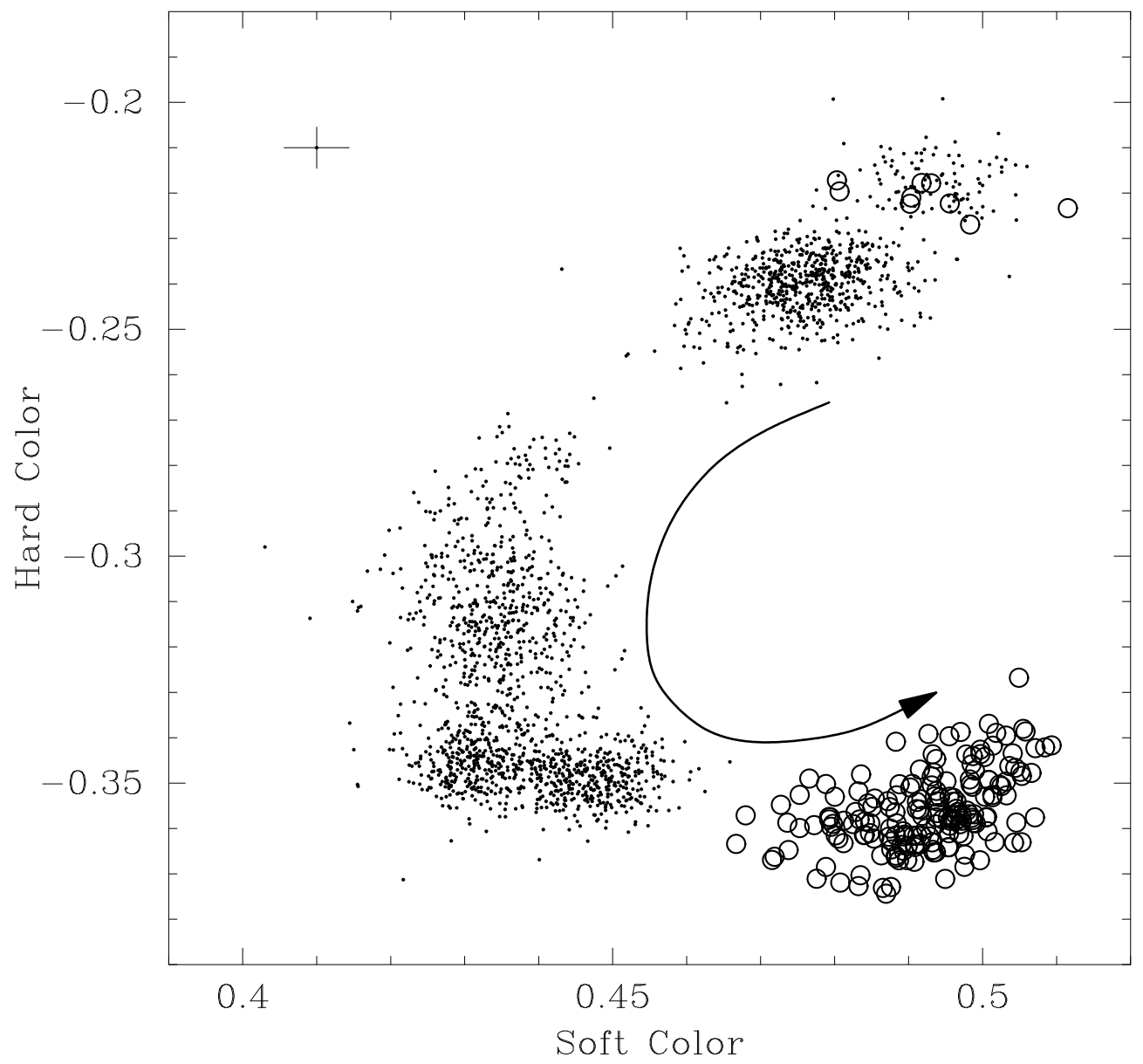

Fig. 2.- Color-color diagram of $4 \mathrm{U}$ 1728-34. The soft and hard colors are defined as (the logarithm of) the ratio of count rates in the bands $3.5-6.4 \mathrm{keV}$ and $2.0-3.5 \mathrm{keV}$, and $9.7-16.0 \mathrm{keV}$ and $6.4-9.7 \mathrm{keV}$, respectively. The contribution of the background has been subtracted, but no dead-time correction was applied to the data (in this case the dead-time effects on the colors are less than 1\%). Each point represents $128 \mathrm{~s}$ of data. Typical error bars are shown. Dots and open circles indicate segments with and without detectable kHz QPOs, respectively. The arrow indicates the sense in which $\dot{M}$ is inferred to increase. 


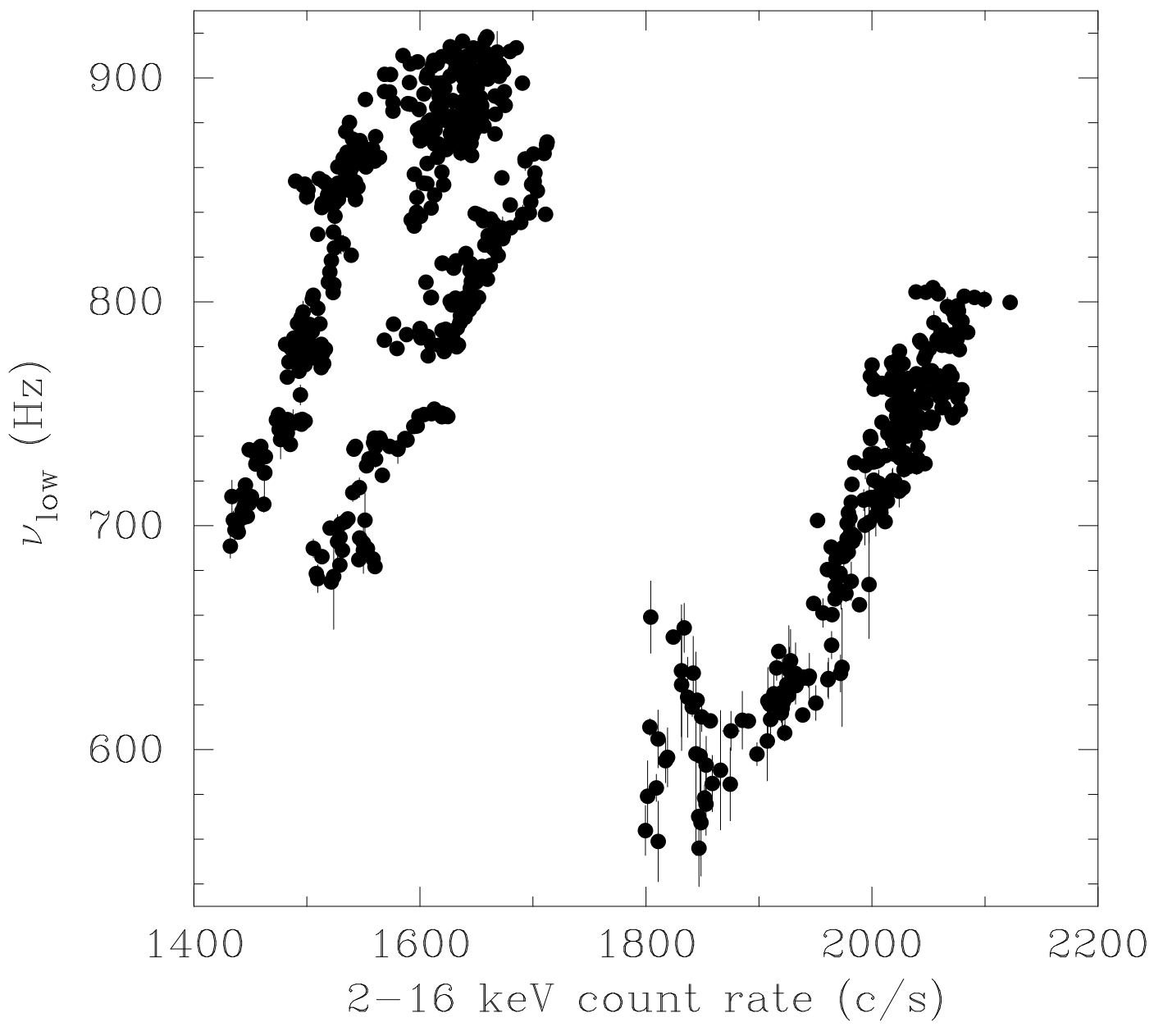

Fig. 3.- Relation between the frequency of the lower $\mathrm{kHz}$ QPO and the $2-16 \mathrm{keV}$ count rate. The count rates have been corrected for background, and normalized to 5 detectors. Each point represents a 128-s segment. We only includes data where both $\mathrm{kHz}$ QPOs were detected simultaneously, so that we can unambiguously identify the lower $\mathrm{kHz}$ peak. 


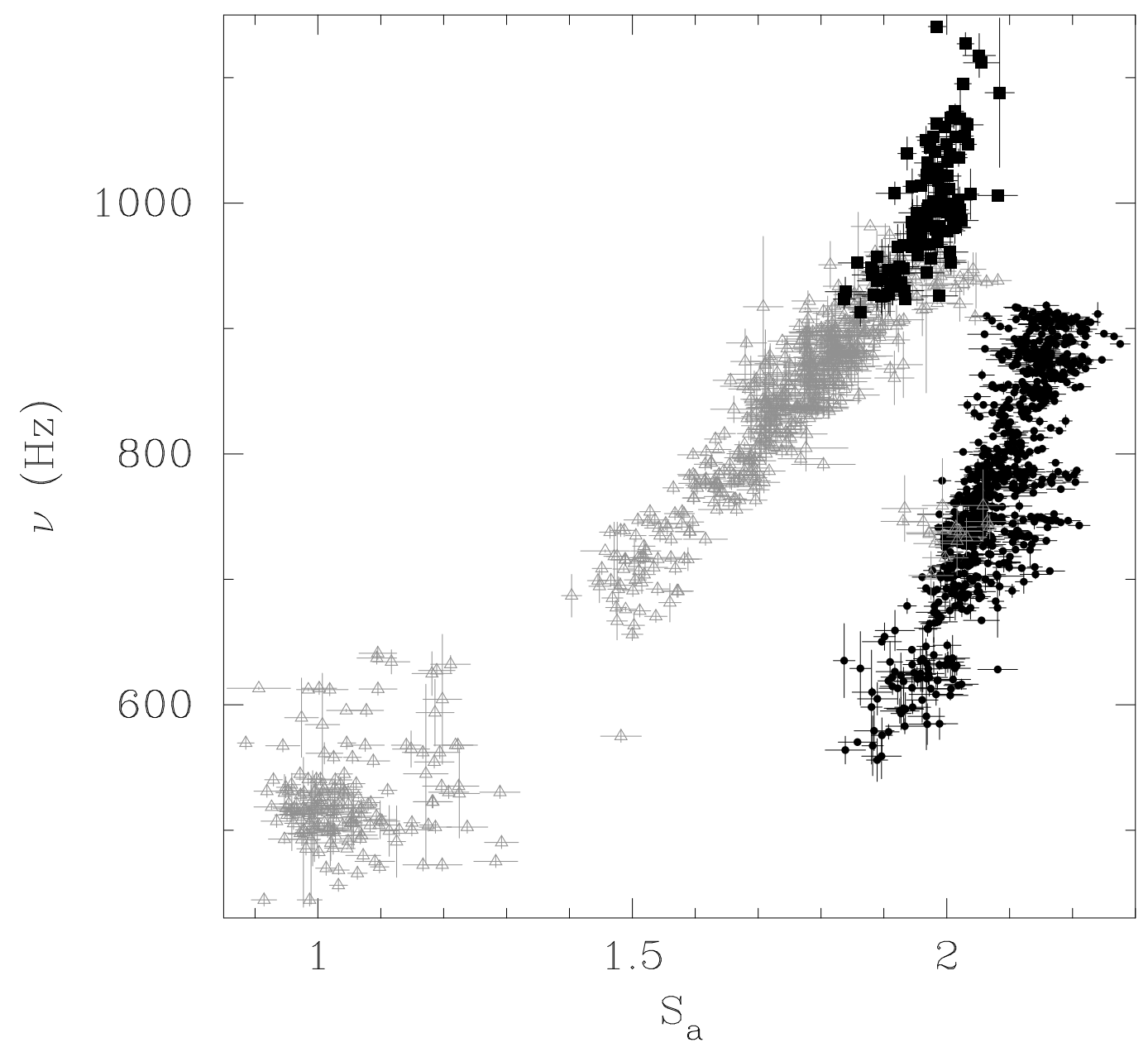

Fig. 4.- Diagram of the frequencies of the upper and lower $\mathrm{kHz}$ QPOs vs. the position of the source on the color-color diagram, as measured by $S_{\mathrm{a}}$ (see Fig. 2, and text). Black circles and squares represent the lower and the upper $\mathrm{kHz}$ peak, respectively. Grey triangles represent segments where we only detected one of the $\mathrm{kHz}$ QPOs in the power spectrum. 